\title{
Thiazolidinedione induces a therapeutic effect on hepatosteatosis by regulating stearoyl-CoA desaturase-1, lipase activity, leptin and resistin
}

\author{
HESSAH MOHAMMED AL-MUZAFAR and KAMAL ADEL AMIN \\ Department of Chemistry and Biochemistry, College of Science, Imam Abdulrahman \\ Bin Faisal University, Dammam 31441, Saudi Arabia
}

Received November 5, 2017; Accepted April 6, 2018

DOI: $10.3892 /$ etm.2018.6563

\begin{abstract}
Hepatosteatosis is a disease present worldwide, which presents a number of health problems. Recently, thiazolidinedione (TZD) has been used as a therapy for lipid disorders. The present study demonstrates the potential of TZD as a treatment for hepatosteatosis and its mechanism of action, particularly focusing on its role in lipid metabolism. A total of 60 (80-90 g) rats were divided into three groups: A normal group with a standard diet, a high-fat, high-carbohydrate diet (HFCD) group or a HFCD+TZD group ( $n=20 /$ group). The HFCD induced hepatosteatosis over a period of 12 weeks and the HFCD+TZD group were administered TZD in weeks 13-16. Blood and tissue samples were collected to measure hepatic function, the lipid profile, metabolism and hormone biomarkers, including serum triglyceride (TG), lipoprotein lipase (LPL), stearoyl-CoA desaturase (SCD-1), leptin and resistin. The HFCD-fed rats exhibited a significant increase in serum TG, total cholesterol, low-density lipoproteins, alanine transaminase and bilirubin compared with the normal group as well as a significant decrease in high-density lipoprotein. In addition, serum leptin and resistin were significantly elevated in the HFCD group compared with the normal group. The administration of TZD significantly increased SCD-1 activity and significantly inhibited LPL activity. It also attenuated the changes in the lipid profiles and normalized serum leptin and resistin levels. The results of the present study indicated that HFCD induced lipid abnormalities associated with hypertriglyceridemia, hypercholesterolemia and hepatosteatosis. These changes resulted from disruption to leptin and resistin, which may be due to alterations in LPL and
\end{abstract}

Correspondence to: Professor Kamal Adel Amin, Department of Chemistry and Biochemistry, College of Science, Imam Abdulrahman Bin Faisal University, 42 Prince Naif Street, Dammam 31441, Saudi Arabia

E-mail: kaothman@uod.edu.sa

Key words: thiazolidinedione, lipid metabolism, stearoyl-CoA desaturase, lipase, high-fat, high-carbohydrate diet, hepatosteatosis
SCD-1 activity. TZD mitigated the effects of HFCD-induced hepatosteatosis, indicating a possible regulatory effect of TZD in the development of hepatosteatosis. The authors suggest that the manipulation of SCD-1 and lipase by TZD may be useful as a treatment for hepatosteatosis.

\section{Introduction}

Hepatosteatosis describes the abnormal buildup of fat within the hepatic cells in the absence of alcohol consumption. It results from an imbalance between uptake, oxidation and transport of fat and may be associated with metabolic dysfunction, obesity and dyslipidemia. Hepatosteatosis may develop into progressive hepatic inflammation (steatohepatitis), fibrosis or cirrhosis $(1,2)$.

The prevalence of hepatosteatosis is increasing globally and it is one of the most common diseases of the liver $(3,4)$. Hepatosteatosis is associated with metabolic syndrome, high alcohol consumption, a poor diet and obesity $(1,5,6)$. The symptoms of hepatosteatosis are often nonspecific, which makes them easy to miss and means that hepatosteatosis can be difficult to diagnose (7). Elevated liver transaminase levels may be a useful diagnostic marker if present, however they often remain within a normal range (8).

Hepatosteatosis may develop into hepatic cirrhosis or hepatocellular carcinoma and may cause the mortality of elderly patients (9). At present the primary method of treating hepatosteatosis/non-alcoholic steatohepatitis (NASH) is adopting a healthy lifestyle, for example, by partaking in regular exercise. However, lifestyle changes are often hard to implement and patients with non-alcoholic fatty liver disease (NAFLD) often have difficulty maintaining an improved lifestyle; therefore, it has been suggested that such patients should be prescribed pharmacological therapy (10).

NAFLDs cover a spectrum of liver conditions extending from simple steatosis, severe steatosis to NASH and can be complicated by fibrosis, cirrhosis, end-period liver failure and hepatocellular carcinoma $(3,4)$. In a majority of cases simple steatosis does not affect the overall survival, while severe steatosis, without inflammation, may affect metabolic function. NASH, an inflammatory disorder, has been associated with increased mortality $(1,7)$. NASH includes steatosis, 
inflammation and hepatocyte ballooning with or without fibrosis $(1,7)$.

Hepatosteatosis increases the risk of cardiovascular disease (CVD) and metabolic syndrome (11). The primary indicator of hepatosteatosis is dyslipidemia, which is characterized by low high-density lipoprotein (HDL) levels, hypertriglyceridemia and a high level of low-density lipoprotein (LDL) (12). Currently thiazolidinedione (TZD), which improves blood lipid profiles, glucose levels and insulin sensitivity is the therapy of choice for type 2 diabetes mellitus (T2DM) (13). TZD is a member of the peroxisome proliferator-activated receptor- $\gamma$ agonists family (PPAR $\gamma$ ), which has been used in clinical trials, either alone or in combination with oral antidiabetic medication to treat patients with T2DM (14).

The molecular, enzymatic and hormonal changes associated with hepatosteatosis and other similar disorders remain unknown. In addition, the method of pathogenesis of hepatosteatosis has not yet been adequately described, although inflammatory cytokines, oxidative stress, leptin and insulin resistance are considered to be significant during its development (2). At present, TZD, an insulin sensitizer and vitamin E, an antioxidant, in combination with lipid-depressing medications are the most promising therapeutic strategies to treat patients with hepatosteatosis/NASH (15). TZD is used to treat hyperglycemia, insulin resistance and T2DM, however its mechanism of action against hepatosteatosis remains unclear. It has been reported that 30-45 mg TZD (commercial name, pioglitazone) significantly improves insulin sensitivity, glycated hemoglobin levels and the lipid profile of patients with T2DM that have abnormal glycemic regulation and slight dyslipidemia (16).

The administration of pioglitazone, which is a type of TZD, has various effects; it increases fat deposition in several types of muscle, perirenal and brown adipose tissue but decreases fat accumulation in hepatic tissue (17). In adipose tissue, increased fat deposition is associated with lipoprotein lipase (LPL) activity, however this is not the case in the muscles (18). Therefore, further investigations into the association between TZD and LPL activity is required.

It has been demonstrated that mice lacking stearoyl-CoA desaturase (SCD-1) exhibit increased lipid oxidation due to the upregulated expression of acyl-CoA oxidase and very long-chain acyl-CoA dehydrogenase and the downregulation of lipid synthesis (19). In addition, SCD-1 is involved in the metabolic response to leptin, a hormone of the fat metabolism, upregulates constituents of insulin signaling and affects the metabolism of glycogen (20). SCD-1 therefore appears to serve a key role in metabolic control and the regulation of its activity may be beneficial in the treatment of hepatosteatosis, obesity, T2DM, cardiovascular disorders and other metabolic illnesses.

It was therefore hypothesized that the expression of SCD-1 promotes lipogenesis and inhibits lipid oxidation, which may induce the accumulation of fat in those consuming a high-calorie diet and potentially lead to hepatosteatosis. High-carbohydrate foods enhance de novo lipogenesis (21); however the effect of TZD on LPL and SCD-1 remains unclear. Previous studies have investigated the association between TZD and lipids; however, the results of such studies are contradictory and the effect on serum triglycerides (TGs) and total cholesterol (TC) and their role exacerbating hepatosteatosis remain unclear $(14,22,23)$. Furthermore, the results of studies investigating the role of TZD in the hormonal and enzymatic control of lipid metabolism are varied and sometimes controversial (23-27). Therefore the present study aimed to explore the lipid metabolism and hormonal regulation associated with hepatosteatosis. In addition, the role of TZD and the possible molecular mechanisms of hepatosteatosis therapy were investigated, as well as the potential connection between hepatosteatosis and SCD-1.

\section{Materials and methods}

Experimental animals. A total of 60 male 6 week-old albino rats, weighing 80-90 g, were obtained from the Institute for Research and Medical Consultation (IRMC; Imam Abdulrahman Bin Faisal University, Dammam, Saudi Arabia). Animals were kept for 1 week prior to the start of the experiment. The rats were kept separately in elastic pens at a temperature of $22 \pm 3^{\circ} \mathrm{C}$, humidity of $40-60 \%$ and a $12-\mathrm{h}$ light/dark cycle in the animal house of the IRMC laboratories. All rats had ad libitum access to food and water.

Diet. Two different types of diet were provided to the rats, either a normal rat chow diet or a high-fat, high-carbohydrate diet (HFCD; Enterprise Grain Company, Kremlin, OK, USA). Normal rat chow pellets were composed of protein ration (whey protein, soya bean and meat), corn, calcium phosphate, calcium carbonate, magnesium oxide, sodium chloride and vitamins. The standard rat food was composed of $60 \%$ carbohydrates, $8 \%$ fat, $22 \%$ basic protein, $10 \%$ dietary fiber, vitamins and minerals. The HFCD contained $65 \%$ carbohydrates, $15 \%$ crude protein, $15 \%$ fat [palmitate and stearate saturated fatty acids (SFAs)] and 5\% dietary fiber, vitamins and minerals. The HFCD was used to develop the rat model of experimental hepatosteatosis and was consistent with the formula used previously by Al-Muzafar and Amin (28).

Chemicals. TZD (pioglitazone hydrogen chloride) was purchased from Santa Cruz Biotechnology, Inc. (Dallas, TX, USA; cat. no. TX75220). This was the only type of TZD used in the present study. A total of $70 \mathrm{mg}$ TZD powder was dissolved in $20 \mathrm{ml}$ distilled water and each rat received $1 \mathrm{ml}$ per oral administration (os) per day at a dose of $17.5 \mathrm{mg} / \mathrm{kg}$ body weight for 4 weeks.

Assay kits. Colorimetric assay kits obtained from HUMAN Gesellschaft für Biochemica und Diagnostica $\mathrm{mbH}$ (Wiesbaden, Germany) were used to assess the serum of rats and determine their lipid profile (TGs, TC, LDL and HDL) and liver function [alanine transaminase (ALT), albumin and bilirubin]. TGs were measured by GPO-PAP method, enzymatic colorimetric test for TG with lipid clearing factor (cat. no. 10724 kit). TC was determined by CHOD-PAP method, enzymatic colorimetric test for cholesterol with lipid clearing factor (cat. no. 10028 kit). LDL was determined by direct homogenous enzymatic colorimetric test (cat. no. 10294). HDL measured using, liquicolor direct homogenous enzymatic test (cat. no. 10284). ALT analyzed by IFCC mod. liquiUV (cat. no. 12022). Albumin was determined by BCG-method, photometric colorimetric test (cat. no. 10560). Bilirubin measured 
by bilirubin Direct/total liquicolor (cat. no. 10740). LDH evaluated by LDH SCE mod. liquiUV (cat. no. 12024). Lipid hormones, leptin and resistin were measured using ELISA kits from Bertin Bioreagent (cat. nos. A051760 and A05179; Montigny le Bretonneux, France). SCD-1 was measured using an ELISA kit (cat. no. MBS923022) obtained from MyBiosource, Inc. (San Diego, CA, USA).

Serum LPL activity was measured using an assay kit (cat. no. 700640) purchased from Cayman Chemical Company (Ann Arbor, MI, USA). The assay used fluorescence to identify serum LPL activity by converting arachidonoyl-1-thioglycerol into thioglycerol and arachidonic acid. The thioglycerol then reacted with the thiol-fluorometric indicator to provide a fluorescent compound, which was measured at 380-390 (excitation) and 510-520 $\mathrm{nm}$ (emission).

Experimental design and animal grouping. The 60 male rats were randomly allocated into 3 groups ( $n=20 /$ group). The first group was administered a standard chow diet throughout the study period (16 weeks). The second group (positive control) was fed HFCD for 16 weeks to induce hepatosteatosis and the third group was fed HFCD and received treatment with TZD (17.5 mg/kg per os) daily between weeks 12 and 16. TZD was administered daily at 8:00 a.m. in a volume equal to $5 \mathrm{ml} / \mathrm{kg}$ at a dose of $17.5 \mathrm{mg} / \mathrm{kg}$. Rats of the TZD-untreated groups received equal volumes $(1 \mathrm{ml})$ distilled water daily at the same time. The total experiment period was 16 weeks, which was divided into the hepatosteatosis induction period (weeks 1-12) and the therapy period (weeks 13-16). Rats were weighed each week, body weights were recorded and weight gains were calculated.

Blood and tissue sampling. Under anesthesia with diethyl ether, blood was collected from the medial canthus of the eye of all rats following overnight fasting at the end of week 16 by using a microhematocrit capillary tube and stored in dry glass centrifuge tubes. The blood was stored at $24 \pm 3^{\circ} \mathrm{C}$ and permitted to coagulate. Samples were centrifuged at $1,400 \mathrm{x} \mathrm{g}$ for $20 \mathrm{~min}$ at a temperature $20^{\circ} \mathrm{C}$ and the clear supernatant serum were stored at $-80^{\circ} \mathrm{C}$ prior to further biochemical analysis. Immediately after blood sampling, the rats were sacrificed by decapitation while still under anesthesia. Part of the liver was extracted, washed with saline and fixed in $10 \%$ formalin at $25^{\circ} \mathrm{C}$ and for $48 \mathrm{~h}$. The tissue samples were embedded in paraffin and cut using a rotary microtome into $3-\mu \mathrm{m}$-thick sections and transferred to slides prior to hematoxylin and eosin staining for histopathological analysis.

Histopathological examinations. Tissue samples underwent hematoxylin and eosin staining. Sections $(3 \mu \mathrm{m})$ were incubated in xylene for $5 \mathrm{~min}$ at $37^{\circ} \mathrm{C}$ followed by $10 \times 10 \mathrm{sec}$ absolute alcohol and running water for $1 \mathrm{~min}$ at $22-25^{\circ} \mathrm{C}$. Sections were incubated with Harris' Hematoxylin for 9 min at $35^{\circ} \mathrm{C}$ and washed with water for $2 \mathrm{~min}$ at $22-25^{\circ} \mathrm{C}$. Differentiation was achieved using $1 \%$ acid alcohol $(2 \times 10 \mathrm{sec})$, followed by $30 \mathrm{sec}$ in $0.2 \%$ ammonia water and running water for $1 \mathrm{~min}$ at $22-25^{\circ} \mathrm{C}$. Prior to microscopic examination, eosin solution was added for $2 \mathrm{~min}$ and sections were washed with water for 1-5 min. and dehydrated in absolute alcohol (10x10 sec) at room temperature. The nucleus was stained blue and the cytoplasm appeared pink. A light microscope was used to inspect the structure and characteristics of hepatic cells, whether fat globules or inflammation were present and whether the hepatocytes had undergone any degenerative alterations. The histological grading system for hepatosteatosis, NAFLD activity score, was used to assess the samples at low (x40) and high (x100) magnification (29). The presence of minor/moderate fat droplets, micro- and macrovesicular hepatosteatosis, hepatolobular inflammation and the presence of inflammatory cells were recorded and used to determine the hepatosteatosis score, as previously described $(28,29)$.

Statistical analysis. The results were analyzed by one-way analysis of variance followed by Tukey-Kramer post-hoc analysis. The data are presented as the mean \pm standard error of the mean. $\mathrm{P}<0.05$ was considered to indicate a statistically significant difference. Statistical examination was performed using GraphPad Prism 6 software (GraphPad Software, Inc., La Jolla, CA, USA).

\section{Results}

HFCD increases body weight and liver function markers. The mean body weight gain was significantly increased in the HFCD group compared with the normal group ( $\mathrm{P}<0.05$; Fig. 1). A significant elevation in the serum total bilirubin and direct bilirubin levels, in addition to LDH and ALT activities as liver function markers was observed in the HFCD group compared with the control $(\mathrm{P}<0.05$; Table I and Fig. 2). The increased levels of bilirubin and ALT activity indicated injuries to the hepatocytes however these changes were ameliorated by the administration of TZD. No significant changes in albumin between the different groups were observed.

HFCD affects the blood lipid profile of rats. A significant increase in serum TG, TC and LDL was observed in the HFCD group compared with the normal group $(\mathrm{P}<0.01$; Fig. 3A-C), whereas there was a significant decrease in serum HDL $(\mathrm{P}<0.01$; Fig. 3D). Treatment with TZD significantly reversed these changes (Fig. 3).

HFCD increases the level of adipose tissue hormones. A significant increase was observed in the level of leptin (Fig. 4A) and resistin (Fig. 4B) in the serum of the HFCD group compared with the normal group $(\mathrm{P}<0.05)$, these changes indicated increased fat accumulation and the development of hepatosteatosis. However, TZD administration recovered the levels of these hormones to similar levels as the normal group.

HFCD leads to an increase in the steatosis score. The steatosis score of the normal group was 0 for $85 \%$ of cases and 1 for $10 \%$ of cases, whereas the HFCD group steatosis score was 1 for $65 \%$ of cases and 2 for $30 \%$ of cases (Table II). This indicates that the steatosis scores were markedly increased in the HFCD group compared with the normal group. The HFCD+TZD group steatosis scores were 0 for $60 \%$ of cases and 1 for $35 \%$ of cases. These results indicate that the administration of TZD markedly reduced the steatosis scores to similar levels as the normal group. 


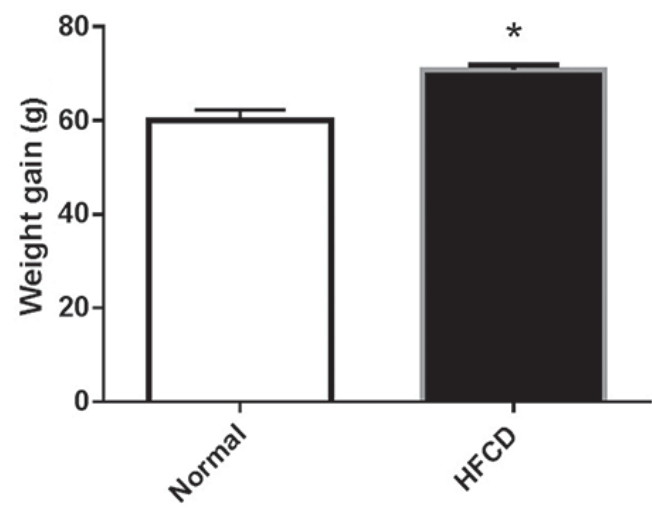

Figure 1. Effect of normal diet or HFCD on rat body weight gain. ${ }^{*} \mathrm{P}<0.05$ vs. normal group. HFCD, HFCD, high-fat, high-carbohydrate diet.

TZD treatment ameliorates the increase in lipid metabolic enzymes caused by HFCD. A notable elevation in the level of SCD-1 was observed in the HFCD group compared with the normal group (Table III) and this was further significantly increased by treatment with TZD. In addition, LPL activity was notably increased in the HFCD group compared with the normal group, however the addition of TZD significantly reversed this increase to a lower level than the normal group.

Treatment with TZD restores normal pathology following a HFCD. Histopathological examination was performed following the administration of a normal, HFCD or TZD+HFCD diet. Ordinary histological configuration (non-visible micro and macrovesicular hepatosteatosis) was observed in the hepatocytes from the normal group (Fig. 5A). However, in the HFCD group several fat droplets, minor to moderate micro and macrovesicular hepatosteatosis, degenerative alterations and focal inflammation were observed in the periportal area of the liver (Fig. 5B). In the HCFD+TZD group, hepatocytes with an ordinary structure were observed (Fig. 5C), indicating that treatment with TZD restored the pathology of the hepatocytes to normal.

\section{Discussion}

The present study revealed that the lipid profile was disrupted and the liver function was impaired in the HFCD group compared with the normal group; the enzymatic control of lipid metabolism (SCD-1 and LPL) was also elevated. In addition, hormones associated with lipid accumulation including, leptin and resistin were increased in the HFCD group compared with the normal group. These results indicate that a variety of biochemical markers are involved in hepatosteatosis pathogenesis.

At present, a complex hypothesis suggests that multiple parallel factors are involved in hepatosteatosis development, these factors include: Obesity; adipose tissue hormones; nutritional factors; gut microbiota; metabolic syndrome, including dyslipidemia, visceral adiposity, increased lipolysis, decreased lipogenesis, hypertriglyceridemia, hyperglycemia, insulin resistance, high BMI and T2DM; age and genetic factors $(30,31)$. However, the primary regulator has yet to be identified. The present study demonstrated that HFCD leads to elevation of the lipid profile (TG and TC) and an increased incidence of metabolic disturbances, which is considered to be a leading cause of hepatosteatosis that may advance to prolonged hepatic disease and hepatic fibrosis (32). This may ultimately result in hepatocellular tumors and hepatic-associated mortality (33).

Chemotherapy is considered an important treatment option as patients suffering from obesity with hepatosteatosis often have difficulty maintaining an improved lifestyle and diet modifications (34). At present, the biochemical and pathological progress of hepatosteatosis and steatohepatitis has not been fully explained. However, lipid metabolism, hormonal and enzymatic regulation, inflammatory markers and oxidative stress are considered to be significant in the initiation and progress of the disorder (30-32). The present study indicated that hepatosteatosis may be associated with moderate to mild lobular infiltration by fat globules, since elevated liver indexes of serum ALT, LDH and bilirubin and an increased lipid profile were observed compared with the control group. The administration of TZD recovered hepatic function in accordance with a previous study by Mazzotti et al (35).

In the present study, TZD resulted in a significantly decreased serum TG level compared with the HFCD group. These results are in agreement with those of Abd El-Haleim et al (36) and also indicate that treatment with TZD has a significant role on the serum lipid profile, as suggested by Wang et al (37). However, it has been previously reported that TZD administration may lead to deteriorating microvesicular steatosis and raised hepatic TG levels, through decreasing blood TG and free fatty acid (23). He et al (38) reported that treatment with TZD had no significant influence on the body mass index, body fat percentage or serum TG and TC levels.

The authors suggest that in the present study, serum TG increased in the HFCD group and accumulated in the liver, while TZD mobilized fat from the hepatic cells for $\beta$-oxidation and caused normal fat deposition/lipogenesis, which led to a return of the serum TG to normal levels. In addition, TZD as an insulin sensitizer serves a major role in returning postprandial lipemia to regular physiological levels and correcting fasting hypertriglyceridemia; thereby protecting against hepatosteatosis and atherogenic risk in T2DM (39). TZD therapy has a favorable effect on numerous lipid types, leading to decreases in TG, TC and LDL serum levels and an increase in HDL levels (40).

The present study revealed a significantly decreased serum cholesterol level in the TZD group compared with the HFCD group, which has also been reported in a number of previous studies $(40,41)$. TZD reduces cholesterol by lowering the key enzyme in cholesterol biosynthesis hydroxymethylglutaryl-CoA reductase mRNA levels and decreasing the intracellular content of cholesterol, which indicates a change in the uptake of cholesterol as well as the inhibition of cholesterol biosynthesis (42). The results of the present study supported this theory as the activity of hepatic LPL was inhibited and the uptake of HDL cholesterol was reduced leading to increase in HDL level in the TZD group. TZD may function as link between lipid and carbohydrate pathways acting as activator for adenosine monophosphate-activated protein kinase, inducing an activation of fatty acid oxidation and decreasing in TG synthesis associated with a state of cellular energy change $(43,44)$. 
Table I. Effect of HFCD and TZD on liver function in rats.

\begin{tabular}{lccc}
\hline Marker & Normal & HFCD & HFCD+TZD \\
\hline Albumin $(\mathrm{g} / \mathrm{dl})$ & $3.12 \pm 0.20$ & $2.99 \pm 1.98$ & $3.62 \pm 0.30$ \\
Total proteins $(\mathrm{g} / \mathrm{dl})$ & $8.41 \pm 0.70$ & $8.39 \pm 0.60$ & $8.11 \pm 0.55$ \\
Total bilirubin $(\mathrm{mg} / \mathrm{dl})$ & $1.42 \pm 0.16$ & $2.36 \pm 0.23^{\mathrm{a}}$ & $1.73 \pm 0.17^{\mathrm{b}}$ \\
Direct bilirubin $(\mathrm{mg} / \mathrm{dl})$ & $0.79 \pm 0.09$ & $2.08 \pm 0.10^{\mathrm{a}}$ & $1.51 \pm 0.13^{\mathrm{b}}$ \\
Lactate dehydrogenase & $621.49 \pm 24.21$ & $825.31 \pm 56.11^{\mathrm{a}}$ & $636.98 \pm 44.48^{\mathrm{b}}$ \\
\hline
\end{tabular}

${ }^{\mathrm{a}} \mathrm{P}<0.05$ vs. the normal group. ${ }^{\mathrm{b}} \mathrm{P}<0.05$ vs. the HFCD group. The data are presented as the mean \pm standard error of the mean. The different superscript letters indicate a significant difference at $\mathrm{P}>0.05$. TZD, thiazolidinedione; HFCD, high-fat, high-carbohydrate diet.

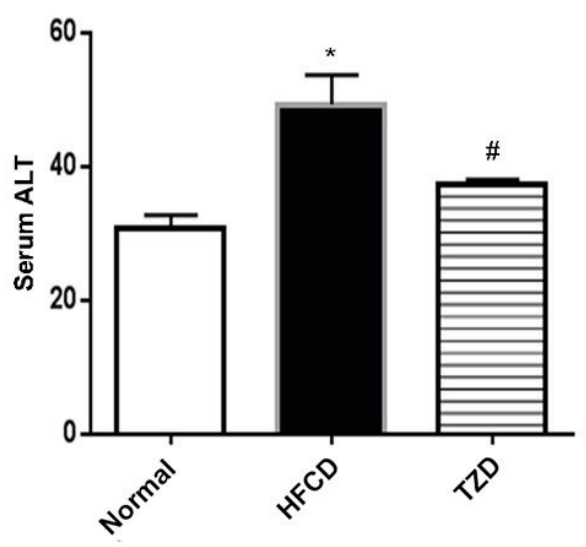

Figure 2. Effect of normal diet, HFCD and HFCD+TZD on ALT as liver function biomarker. ${ }^{*} \mathrm{P}<0.05$ vs. normal group. ${ }^{\#} \mathrm{P}<0.05$ vs. HFCD group. HFCD, high-fat, high-carbohydrate diet; TZD, thiazolidinedione.

In summary, TZD exhibited a direct effect on the liver and confirming these findings in humans may clarify the significant progresses in blood lipids that detected with TZD treatment in diabetic patients (42).

At present, TZD as an insulin sensitizer and vitamin $\mathrm{E}$ as an antioxidant are the most favorable treatment options for NAFLD and NASH as fat-lowering medications (15). Each has an optimal result on aminotransferase, inflammation and fat accumulation. Vitamin E may recover normal histology in hepatosteatosis but safety issues limit its use $(15,45)$. However, other medications, including ursodeoxycholic acid, statins, metformin, orlistat and pentoxifylline only have moderately positive results $(6,10,15)$.

The current study reported that hormones and enzymes regulating lipid metabolism serve a role in the pathogenesis and progression of hepatosteatosis in rats and that TZD may act as a modifier of the lipid profile, hormones and enzymatic control for lipid metabolism for the prevention and treatment of fatty liver induced by a HFCD. This result is supported by $\mathrm{Xu}$ et al (5) who used TZD at $4 \mathrm{mg}$ for 8 weeks in HFCD rats. TZD may mitigate insulin resistance as well as histological injury and biochemical markers in high fat-induced fatty livers in rats as reported by Mohan et al (46) and Peng et al (23).

The present study revealed a significantly elevated resistin level in the HFCD group, while the administration of TZD reversed this increase. The resistin hormone (resistance to insulin) was initially identified in mice in 2001 and was named for its capacity to interfere with insulin action (47). Resistin was originally defined as an adipocyte-specific hormone and it has been suggested as a link between obesity, insulin resistance, diabetes and NAFLD (48).

Novel pharmacological agents that decrease the level of resistin have been identified, which prevent the adverse effect of resistin on the lipid metabolism and provide a therapeutic strategy for the treatment of hepatic injuries $(49,50)$. The mechanism of action of TZD against lipid metabolism is considered to be through decreasing resistin, which reduces any interference with the action of insulin and stimulates lipogenesis. The reducing effect of TZD in blood resistin was associated with the reduction in liver fat content and improvement in sensitivity of insulin (51). In addition TZD attenuates insulin resistance through adiponectin-dependent and -independent signaling pathways $(49,50)$. The use of TZD to control leptin and liver enzymes represents a novel approach for hepatosteatosis therapy as presented in the current study.

The present study revealed that serum leptin was significantly increased in the HFCD group compared with the normal group. The recognition of the leptin hormone provided a molecular association to obesity and NAFLD similar to effect observed for resistin. Leptin regulates energy homeostasis and is known as the essential mediator within the endocrine system (52). The data demonstrating increases in serum leptin levels agree with previous findings (53-55). Administration of leptin resulted in elevated energy expenditure, hypophagia and loss of weight, whereas leptin deficiency exhibited decreased energy expenditure, hyperphagia and neuroendocrine axis suppression $(54,55)$. The majority of obese individuals and those with NAFLD are leptin resistant, which presents as high serum leptin levels without controlling energy hemostasis (56). However Canbakan et al (57) reported that leptin levels were elevated in NAFLD and exhibited a preventive effect of leptin against progressive liver damage Further understanding of the molecular mechanisms of the leptin signaling pathway is required to improve the therapeutic options for hepatosteatosis and metabolic syndrome. In the normal rat group, the effects of leptin may be due to its anorectic role and facilitate specific metabolic responses, comprising effective depletion of triacylglycerol from hepatic and other peripheral tissues thereby maintaining healthy liver tissue (58).

Leptin is a lipid metabolic hormone that regulates energy homeostasis and is considered as the principal mediator in the 
A

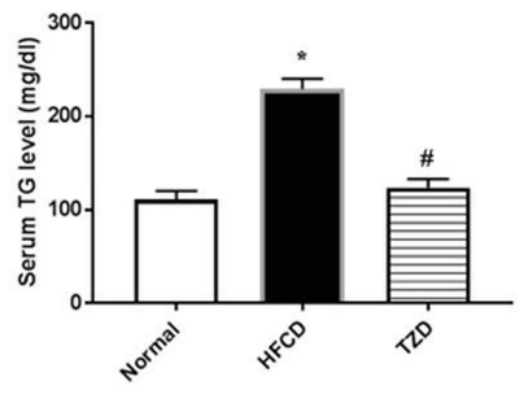

C

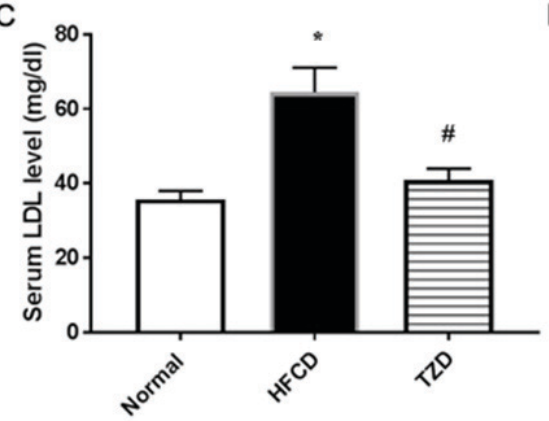

$\mathrm{B}$

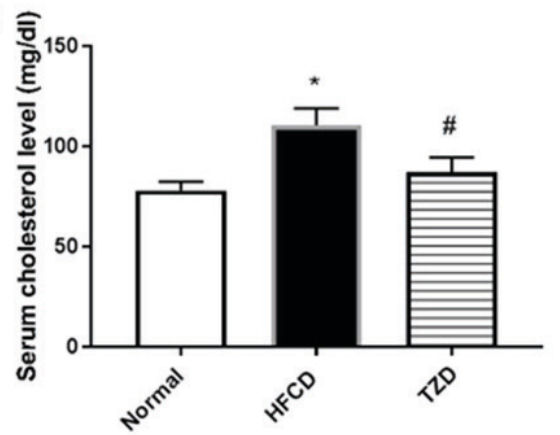

D

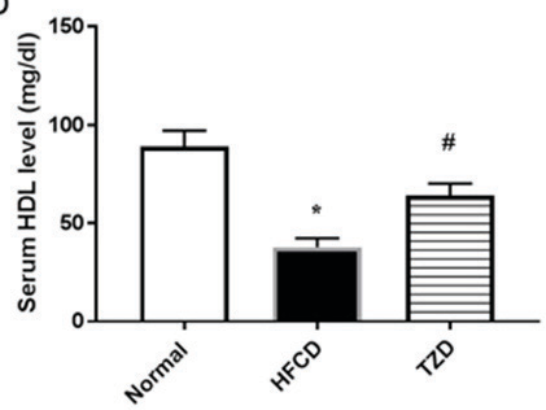

Figure 3. Effect of normal diet, HFCD or HFCD+TZD on serum (A) TG, (B) TC, (C) LDL and (D) HDL levels. "P<0.05 vs. normal group. "P<0.05 vs. HFCD group. HFCD, high-fat, high-carbohydrate diet; TZD, thiazolidinedione; TG, triglycerides; TC, total cholesterol; LDL, low-density lipoprotein.

A

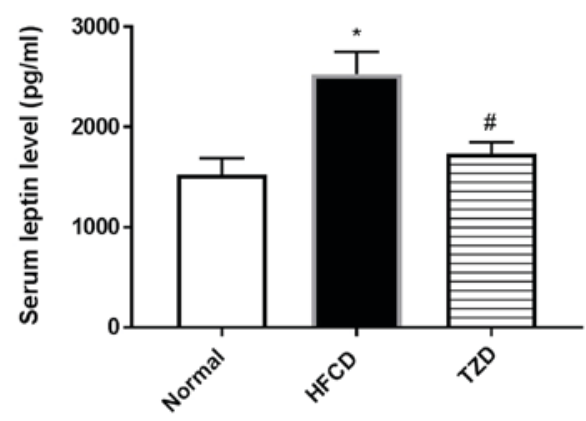

B

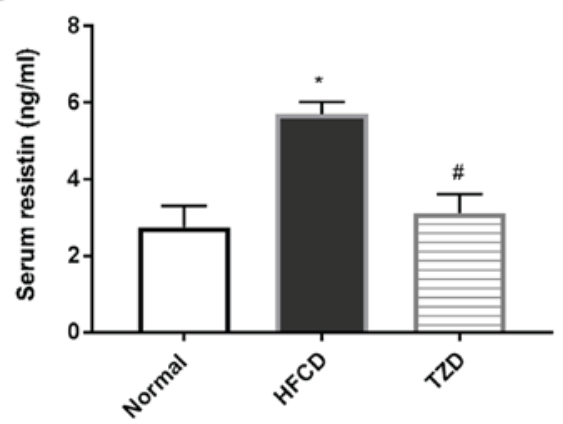

Figure 4. Effect of normal diet, HFCD and HFCD+TZD on (A) leptin and (B) resistin hormone in rats. ${ }^{*} \mathrm{P}<0.05$ vs. normal group. ${ }^{\text {"}} \mathrm{P}<0.05$ vs. HFCD group. HFCD, high-fat, high-carbohydrate diet; TZD, thiazolidinedione.

negative feedback loop (52). In addition, leptin depletes fat from hepatic and other peripheral tissues under normal conditions $(52,59)$. In the current study, HFCD induced elevated leptin and resistin levels, associated with insulin-resistance, and a non-significant elevation in SCD. SCD levels were not sufficient to induce significant conversion of SFA to mono unsaturated fatty acids (MUSFA), causing elevated TGs, accumulation of fat globules in the hepatocytes and the development of hepatosteatosis. However, STZ reverted these changes by decreasing leptin and resistin levels and inducing a significant increase in SCD-1, consequently mobilizing fat from the liver.

During the development of hepatosteatosis, hypertriglyceridemia raises the free fatty acids (FFA) in the blood, which induces insulin resistance; this frequently occurs in obese patients $(38,39)$. The pharmacological basis for the effects of TZD depends on its ability to reduce hypertriglyceridemia and serum FFAs by inhibition of lipolysis and enhancing oxidation, re-esterification and uptake from the blood and hepatic cells (60). The present study investigated whether TZDs regulate lipolysis (the process of monitoring the hydrolysis of TG and efflux of FFA to the blood stream). Inhibition of FFA efflux and lipolysis in the HFCD+TZD group may describe the underlying mechanism for hepatosteatosis treatment by TZDs. He et al (60) proposed that TZD stimulated Akt signaling to lower the cyclic adenosine monophosphate (cAMP) level and thereby decrease the activity of LPL, consequently preventing lipolysis and resulting in reduced hypertriglyceridemia. In the current study, the response of hepatosteatosis to TZD therapy was associated with inhibiting LPL activity; therefore, TZD treatment may have potentially beneficial properties for the blood lipid profile (TG, TC and LDL).

In recent years the frequency of metabolic syndrome has increased and it is typically linked with hepatosteatosis, particularly diabetes, hypertension and cardiovascular disease (61). The results of the present study demonstrated 
Table II. Hepatosteatosis score in the differently treated groups of rats.

\begin{tabular}{lccc}
\hline $\begin{array}{l}\text { Hepatosteatosis } \\
\text { score }(\%)^{\mathrm{a}}\end{array}$ & $\begin{array}{c}\text { Normal } \\
(\%)\end{array}$ & $\begin{array}{c}\text { HFCD } \\
(\%)\end{array}$ & $\begin{array}{c}\text { HFCD+TZD } \\
(\%)\end{array}$ \\
\hline $0(\leq 5)$ & 85 & 0 & 60 \\
$1(6-33)$ & 10 & 65 & 35 \\
$2(34-66)$ & 5 & 30 & 5 \\
$3(\geq 67)$ & 0 & 5 & 0 \\
\hline
\end{tabular}

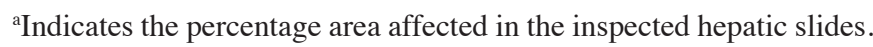
TZD, thiazolidinedione; HFCD, high-fat, high-carbohydrate diet.

that serum leptin and SCD-1 concentration increased in the HFCD group compared with the control group. SCD-1 is a vital metabolic regulator for body weight and is considered as the rate-limiting lipogenic enzyme catalyzing the anabolism of MUSFAs) $(20,62)$, primarily palmitoleate (C16:1) and oleate (C18:1), which describe the majority of fatty acids in TGs, esters, phospholipids and cholesterol esters (20). In addition, SCD-1 is predominantly located in the endoplasmic reticulum, where it undergoes a quick turnover in response to diverse hormonal and nutritional signals (63).

Chong et al (64) reported that activation of SCD-1 and de novo lipogenesis in hepatic and adipose tissue was initiated after high carbohydrate feeding for a short period (3 days). The enzymatic activity of SCD-1 was raised $>7$-fold in livers of non-treated $o b / o b$ mice compared with the wild-type (65). Concentrations of hepatic MUSFAs (C16:1 and C18:1), which are SCD-1 products, were increased in $o b / o b$ mice and normalized by treatment with leptin for 12 days (65). Changes in SCD-1 levels in obese mice decreased body adiposity and increased energy expenditure and insulin sensitivity and induced resistance to a obesity induced by diet (66). Elevated levels of SCD-1 have further been established in insulin-resistant animals and humans (67), which are conditions associated with hepatosteatosis.

The present study suggests that non-significant increases of SCD-1 compared with normal levels affected the MUFA/SFA ratio by increasing lipid profile, high lipogenesis and inducing of steatosis. In addition, palmitate, contained in HFCD, may induce lipogenesis by activating $\mathrm{X}$-Box binding protein-1-mediated endoplasmic reticulum stress, demonstrated by the increase in steatosis score $\geq 2$, associated with blood palmitate $(68,69)$. In the normal rat group, the physiological level of SCD-1 maintained a balanced ratio of MUSFA/SFA to keep the hepatic tissue healthy without fat accumulation. However, in the HFCD group the activity of SCD-1 did not significantly alter and subsequent changes in MUSFA may not meet the threshold target for observing further effects. TZD therapy may elevate the activity of SCD-1 and maintain threshold levels of MUSFA to treat NAFLD.

In recent years, evidence has indicated that SCD-1 overexpression is associated with liver fibrosis and hepatocellular carcinoma $(68,70)$. In addition, a high fructose diet prolonged hepatic SCD-1 activation and promoted TG accumulation and steatosis $(71,72)$. It has also been determined that elevated SCD-1 is associated with obesity (73). However, other studies have identified no significant correlation between muscle or adipose SCD-1 mRNA and insulin sensitivity or body mass index (24).

In downregulated SCD-1 mice a number of genes associated with lipid synthesis are downregulated, whereas genes associated with lipid oxidation are upregulated (25). This suggests that a consequence of reduced SCD-1 is the activation of fat oxidation and decreased triacylglycol biosynthesis loading and storage (25). Consistent with this observation, a lower SCD-1 index protects against diet-induced obesity (53), which appears to be consistent with the level of the normal group compared with the HFCD and TZD groups in the present study.

The non-significant notable elevated SCD-1 observed in the HFCD group compared with the normal group may be due to the conversion of SFA into unsaturated (U)SFA, which is important for the clearance of solid fat by conversion into miscible fatty acids. The activity of SCD-1 prevents the accumulation of palmitate via the conversion into USFA $(19,74)$. The authors suggest that when the SFA/MUSFA ratio is increased, it favors the saturated type. As the fatty component of HFCD was primarily composed of palmitate and stearate SFA, it resulted in the accumulation of TG in hepatic tissues. The ratio of SFA to MUSFA also affects the composition of phospholipids (19). In addition, variation in this ratio has been involved in several disease states involving obesity, cardiovascular disease, diabetes, neurological illness and cancer. Therefore SCD-1 expression is of physiological importance in disease and normal conditions (19). The high leptin and SCD-1 levels observed in HFCD group of the current study may link to protection against steatosis in response to HFCD or induction of steatosis and the development of steatosis. Furthermore, the occurrence of leptin-resistance in HFCD or obese cases may affect the results.

Elevated hepatic de novo lipogenesis due to dietary sugar is involved in the pathophysiology of hepatosteatosis $(75,76)$. SFA are the end product of de novo lipogenesis and perform lipotoxic roles that stimulate the accumulation of fat in the liver. Desaturation of these SFA by SCD-1 can avoid these harmful effects (74). De novo lipogenesis is associated with hepatosteatosis with a nutritional status rich in monosaccharides and it is proposed that individual hepatic SCD-1 activity is negatively correlated with hepatic lipid aggregation under lipogenic nutritional situations (77). The current study indicated that SCD-1 may be an essential factor in the pathogenesis of HFCD induced steatosis.

TZD may control SCD-1, which catalyzes a rate-regulating step in the synthesis of unsaturated oleic acid, by catalyzing the desaturation of stearic acid (20). The ratio of stearic to oleic acid is associated with the control of cell growth and differentiation via effects on signal transduction and cell membrane flexibility $(18,24)$.

A previous study reported that SCD-1 was activated in hepatosteatosis and consequently its suppression was a potential treatment option (78). SCD-1 deficiency and its inhibitor were considered important for the prevention of steatosis and other metabolic diseases (79). The present study investigated the treatment of hepatosteatosis with TZD and identified an 
Table III. Effect of HFCD and TZD on lipid metabolic enzymes in the differently treated groups of rats.

\begin{tabular}{lrrr}
\hline Enzyme & \multicolumn{1}{c}{ Normal } & HFCD & HFCD+TZD \\
\hline Stearoyl-CoA desaturase $(\mathrm{ng} / \mathrm{ml})$ & $9.0 \pm 1.40$ & $12.0 \pm 1.39$ & $18.0 \pm 1.90^{\mathrm{a}, \mathrm{b}}$ \\
Lipoprotein lipase $(\mathrm{nmol} / \mathrm{min} / \mathrm{ml})$ & $39.14 \pm 3.80$ & $49.61 \pm 9.60$ & $24.50 \pm 2.70^{\mathrm{a}, \mathrm{b}}$ \\
\hline
\end{tabular}

${ }^{\text {aP }}<0.05$ vs. the normal group. ${ }^{\text {b }}<0.05$ vs. the HFCD group. The data are presented as the mean \pm standard error of the mean. TZD, thiazolidinedione; HFCD, high-fat, high-carbohydrate diet.
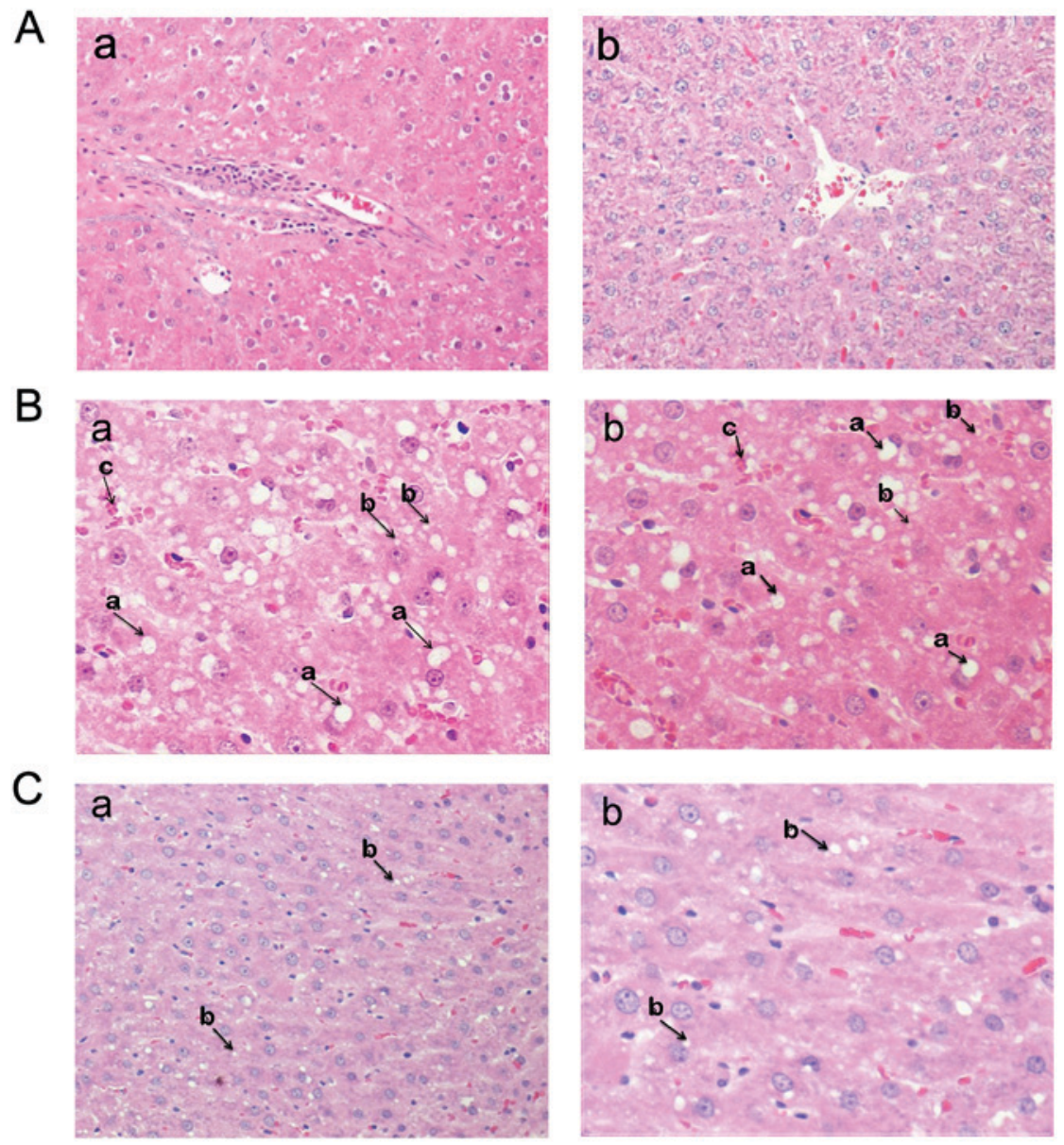

Figure 5. Histopathological examination of different treatment groups. Histopathological outcomes in the (A) normal, (B) HFCD and (C) HFCD+TZD treatment groups. The normal tissue samples ( $\mathrm{Aa}$ ) and $(\mathrm{Ab})$ demonstrated characteristic histological configuration of ordinary hepatocytes, without inflammatory cells in the perivenular area. Several fat bubbles were observed among and inside the hepatocytes in the $(\mathrm{Ba})$ and $(\mathrm{Bb})$ HFCD liver tissue samples, which is associated with deteriorating alterations in the hepatocytes (magnification, x100). The HFCD group had macrovesicular hepatosteatosis (large fat drops present in the hepatocytes, indicated by arrow a) and microvesicular steatosis (slight fat droplets in the hepatocytes, indicated with arrow b). Liver focal periportal inflammation (magnification, x100) and accumulation (cluster) of inflammatory cells in the HFCD group indicated with arrow c. The hepatocytes appeared normalized and had a normal shape and minor lipid globules in the (Ca) and (Cb) HFCD+TZD liver tissue samples. HFCD, high-fat, high-carbohydrate diet; TZD, thiazolidinedione.

alternative mechanism, which was supported by a number of studies using TZD in vitro and in vivo $(18,24)$.

The results revealed that SCD-1 concentration notably increased in the HFCD group compared with the control group. However, treatment with TZD increased its level significantly compared with HFCD group. The authors suggest that the ratio of SFA/USFF is an important factor in the effect of TZD. SCD-1 is associated with pioglitazone (TZD) administration in humans and a 2-fold elevation of SCD-1 mRNA and protein is observed in fat tissue (24).
Pioglitazone also raised the level of SCD-1 in vitro (24). These changes suggest that PPAR $\gamma$ upregulation of SCD-1 resulted in elevated lipogenesis and strengthened adiponectin signaling. Further studies indicated that PPAR $\gamma$ agonists weaken palmitate-induced endoplasmic reticulum stress and apoptosis by SCD-1 generation (80-82). SCD-1 upregulation may lead to the lessening of cardiovascular disturbances through therapy with PPAR $\gamma$ agonists (81).

TZD significantly upregulated the activity of SDC-1, leading to the conversion of inert saturated palmitate into 
reactive monounsaturated acid, which consequently increased its ability to be incorporated in cell membrane phospholipids $(24,81,83)$. In this respect, the degree of unsaturation in membrane phospholipids serves a vital role in numerous membrane functions and irregular incorporation of saturated phospholipids may lead to stiffening and loss function (84). In addition, the conversion increased the likelihood of fatty acid movement and its reaction with cholesterol to form cholesterol esters (85). These events prevent the formation of inert fat globules and mobilize the accumulated fat droplets in the liver compared with the HFCD group. In the TZD group, higher activity of SCD1 favor the MUSFA/SFA ratio. TZD raised the composition of delta 9-cis USFA as (C14:1) myristoleic acid, (C16:1) palmitoleic acid, (C18:1) oleic acid, and (C18:2) linoleic acid, however, TZD decreased the composition of SFA, (C14:0) myristic acid, (C16:0) palmitic acid and (C18:0) stearic acid. These changes resulted in the formation of phospholipids and the mobilization of TG from the accumulated fat in hepatosteatosis and fatty liver, however these findings require further research. Toyama et al (26) demonstrated that hepatic or serum SCD-1 activity and the mRNA level of SCD-1 were not altered by TZD treatment and no variations have been detected for the activity index of SCD-1 in cells treated with TZD (86). TZD may maintain the leptin level resulting in the activation and promotion of SCD-1.

In conclusion, HFCD induced-steatosis resulted in lipid metabolism disorders including hypertriglyceridemia and hypercholesterolemia, which are hormonally controlled by leptin and resistin. Enzymatic changes were also observed in the activity of SCD-1 and lipase. The administration of TZD served a beneficial role in lipid metabolism by normalizing the level of serum TG, TC, leptin and resistin by increasing the level of SCD-1 and suppressing LPL activity. TZD appeared to increase the lipogenesis of oleic USFA and thereby prevented the effect of hepatosteatosis.

The mechanism of action of TZD was inhibition of lipolysis (blood TG hydrolysis and FFA efflux), which occurred by the inhibition of LPL activity. This mechanism may present a pharmacological basis for using TZDs as a hepatosteatosis therapy. The results of the present study suggest that SCD-1 is an essential component in the control of fat metabolism and the clinical use of SCD-1 may be an effective therapy against hepatosteatosis and other constituents of metabolic syndrome, including diabetes and obesity.

\section{Acknowledgements}

The authors thank the histopathological unit of King Fahd Hospital for their support and Dr Awadia S. Awadalla from the College of Medicine and Prof. Dr. Ebtesam A. Al-Suhaimi from the Institute for Research and Medical Consultations at the Imam Abdulrahman bin Faisal University for their cooperation in this study.

\section{Funding}

The present study was financed by a grant from the Deanship of Scientific Research at the University of Imam Abdulrahman Bin Faisal (grant no. 2014082).

\section{Availability of data and materials}

All data produced or analyzed during this study are included in this published article.

\section{Authors' contributions}

KA and HA designed the study, performed the experiments, analyzed and interpreted the data. KA and HA drafted and revised the manuscript. All authors read and approved the final manuscript.

\section{Ethics approval and consent to participate}

The animal experiments were performed in accordance with the National Institutes of Health guidelines for the care and use of laboratory animals and with local ethical guidelines. The study was approved by Imam Abdulrahman Bin Faisal University's Institutional Review Board (approval no. IRB-b-2014-9066).

\section{Patient consent for publication}

Not applicable.

\section{Competing interests}

The authors declare that they have no competing interests.

\section{References}

1. Noureddin M, Mato JM and Lu SC: Nonalcoholic fatty liver disease: Update on pathogenesis, diagnosis, treatment and the role of S-adenosylmethionine. Exp Biol Med (Maywood) 240: 809-820, 2015.

2. Tarantino G,SavastanoS and Colao A:Hepatic steatosis, low-grade chronic inflammation and hormone/growth factor/adipokine imbalance. World J Gastroenterol 16: 4773-8783, 2010.

3. Younossi ZM, Koenig AB, Abdelatif D, Fazel Y, Henry L and Wymer M: Global epidemiology of nonalcoholic fatty liver disease-Meta-analytic assessment of prevalence, incidence, and outcomes. Hepatology 64: 73-84, 2016.

4. Araújo AR, Rosso N, Bedogni G, Tiribelli C and Bellentani S: Global epidemiology of non-alcoholic fatty liver disease/non-alcoholic steatohepatitis: What we need in the future. Liver Int 38 (Suppl 1): S47-S51, 2018.

5. Xu P, Zhang XG, Li YM, Yu CH, Xu L and Xu GY: Research on the protection effect of pioglitazone for non-alcoholic fatty liver disease (NAFLD) in rats. J Zhejiang Univ Sci B 7: 627-633, 2006.

6. Hardy T, Anstee QM and Day CP: Nonalcoholic fatty liver disease: New treatments. Curr Opin Gastroenterol 31: 175-183, 2015.

7. Abd El-Kader SM and El-Den Ashmawy EM: Non-alcoholic fatty liver disease: The diagnosis and management. World J Hepatol 7: 846-858, 2015.

8. Lomonaco R, Chen J and Cusi K: An endocrine perspective of nonalcoholic fatty liver disease (NAFLD). Ther Adv Endocrinol Metab 2: 211-225, 2011.

9. Starley BQ, Calcagno CJ and Harrison SA: Nonalcoholic fatty liver disease and hepatocellular carcinoma: A weighty connection. Hepatology 51: 1820-1832, 2010.

10. Gitto S, Vitale G, Villa E and Andreone P: Treatment of nonalcoholic steatohepatitis in adults: Present and future. Gastroenterol Res Pract 2015: 732870, 2015.

11. Wójcik-Cichy K, Koślińska-Berkan E and Piekarska A: The influence of NAFLD on the risk of atherosclerosis and cardiovascular diseases. Clin Exp Hepatol 4: 1-6, 2018.

12. Sun DQ, Liu WY, Wu SJ, Zhu GQ, Braddock M, Zhang DC, Shi KQ, Song D and Zhen MH: Increased levels of low-density lipoprotein cholesterol within the normal range as a risk factor for nonalcoholic fatty liver disease. Oncotarget 7: 5728-5737, 2016. 
13. Davidson MA, Mattison DR, Azoulay L and Krewski D: Thiazolidinedione drugs in the treatment of type 2 diabetes mellitus: Past, present and future. Crit Rev Toxicol 48: 52-108, 2018.

14. Betteridge DJ: Effects of pioglitazone on lipid and lipoprotein metabolism. Diabetes Obes Metab 9: 640-647, 2007.

15. Takahashi Y, Sugimoto K, Inui H and Fukusato T: Current pharmacological therapies for nonalcoholic fatty liver disease/nonalcoholic steatohepatitis. World J Gastroenterol 21: 3777-3785, 2015.

16. Herz M, Johns D, Reviriego J, Grossman LD, Godin C, Duran S, Hawkins F, Lochnan H, Escobar-Jiménez F, Hardin PA, et al: A randomized, double-blind, placebo-controlled, clinical trial of the effects of pioglitazone on glycemic control and dyslipidemia in oral antihyperglycemic medication-naive patients with type 2 diabetes mellitus. Clin Ther 25: 1074-1095, 2003.

17. de Souza CJ, Eckhardt M, Gagen K, Dong M, Chen W, Laurent D and Burkey BF: Effects of pioglitazone on adipose tissue remodeling within the setting of obesity and insulin resistance. Diabetes 50: 1863-1871, 2001 .

18. Ochiai M and Matsuo T: Pioglitazone-induced increase in the stearoyl-CoA desaturation index and fat accumulation in rat muscles are not related to lipoprotein lipase activity. J Oleo Sci 62: 745-754, 2013

19. Ntambi JM and Miyazaki M: Regulation of stearoyl-CoA desaturases and role in metabolism. Prog Lipid Res 43: 91-104, 2004.

20. Paton CM and Ntambi JM: Biochemical and physiological function of stearoyl-CoA desaturase. Am J Physiol Endocrinol Metab 297: E28-E37, 2009.

21. Marques-Lopes I, Ansorena D, Astiasaran I, Forga L and Martínez JA: Postprandial de novo lipogenesis and metabolic changes induced by a high-carbohydrate, low-fat meal in lean and overweight men. Am J Clin Nutr 73: 253-261, 2001.

22. Jia C, Huan Y, Liu S, Hou S, Sun S, Li C, Liu Q, Jiang Q, Wang Y and Shen Z: Effect of chronic pioglitazone treatment on hepatic gene expression profile in obese C57BL/6J Mice. Int J Mol Sci 16: 12213-1229, 2015.

23. Peng J, Huan Y, Jiang Q, Sun SJ, Jia CM and Shen ZF: Effects and potential mechanisms of pioglitazone on lipid metabolism in obese diabetic KKAy Mice. PPAR Res 2014: 538183, 2014.

24. Yao-Borengasser A, Rassouli N, Varma V, Bodles AM, Rasouli N Unal R, Phanavanh B, Ranganathan G, McGehee RE Jr and Kern PA: Stearoyl-coenzyme A desaturase 1 gene expression increases after pioglitazone treatment and is associated with peroxisomal proliferator-activated receptor-gamma responsiveness. J Clin Endocrinol Metab 93: 4431-4439, 2008.

25. Ntambi JM, Miyazaki M, Stoehr JP, Lan H, Kendziorski CM, Yandell BS, Song Y, Cohen P, Friedman JM and Attie AD: Loss of stearoyl-CoA desaturase-1 function protects mice against adiposity. Proc Natl Acad Sci USA 99: 11482-18486, 2002.

26. Toyama T, Kudo N, Hibino Y, Mitsumoto A, Nishikawa M and Kawashima Y: Effects of pioglitazone on stearoyl-CoA desaturase in obese Zucker fa/fa rats. J Pharmacol Sci 104: 137-145, 2007.

27. Boutari C, Perakakis N and Mantzoros CS: Association of adipokines with development and progression of nonalcoholic fatty liver disease. Endocrinol Metab (Seoul) 33: 33-43, 2018.

28. Al-Muzafar HM and Amin KA: Probiotic improves fatty liver disease by virtue of its action on lipid profiles, leptin, and inflammatory biomarkers. BMC Complement Altern Med 17: 43, 2017.

29. Liang W, Menke AL, Driessen A, Koek GH, Lindeman JH, Stoop R, Havekes LM, Kleemann R and van den Hoek AM: Establishment of a general NAFLD scoring system for rodent models and comparison to human liver pathology. PLoS One 9: e115922, 2014

30. Mikolasevic I, Milic S, Turk Wensveen T, Grgic I, Jakopcic I, Stimac D, Wensveen F and Orlic L: Nonalcoholic fatty liver disease-A multisystem disease? World J Gastroenterol 22: 9488-9505, 2016.

31. Marino L and Jornayvaz FR: Endocrine causes of nonalcoholic fatty liver disease. World J Gastroenterol 1: 11053-11076, 2015.

32. Tilg $\mathrm{H}$ and Moschen AR: Evolution of inflammation in nonalcoholic fatty liver disease: The multiple parallel hits hypothesis Hepatology 52: 1836-1846, 2010.

33. Pang Y, Kartsonaki C, Turnbull I, Guo Y, Clarke R, Chen Y, Bragg F, Yang L, Bian Z, Millwood IY, et al: Diabetes, plasma glucose and incidence of fatty liver, cirrhosis and liver cancer: A prospective study of 0.5 million people. Hepatology 2018 (Epub ahead of print).
34. Zelber-Sagi S, Godos J and Salomone F: Lifestyle changes for the treatment of nonalcoholic fatty liver disease: A review of observational studies and intervention trials. Therap Adv Gastroenterol 9: 392-407, 2016.

35. Mazzotti A, Caletti MT, Marchignoli F, Forlani G and Marchesini G: Which treatment for type 2 diabetes associated with non-alcoholic fatty liver disease? Dig Liver Dis 49: 235-240, 2017.

36. Abd El-Haleim EA, Bahgat AK and Saleh S: Effects of combined PPAR- $\gamma$ and PPAR- $\alpha$ agonist therapy on fructose induced NASH in rats: Modulation of gene expression. Eur J Pharmacol 773: 59-70, 2016.

37. Wang G, Wang X, Zhang Q and Ma Z: Response to pioglitazone treatment is associated with the lipoprotein lipase S447X variant in subjects with type 2 diabetes mellitus. Int J Clin Pract 61: 552-557, 2007.

38. He L, Liu X, Wang L and Yang Z: Thiazolidinediones for nonalcoholic steatohepatitis: A meta-analysis of randomized clinical trials. Medicine (Baltimore) 95: e4947, 2016.

39. Al Majali K, Cooper MB, Staels B, Luc G, Taskinen MR and Betteridge DJ: The effect of sensitisation to insulin with pioglitazone on fasting and postprandial lipid metabolism, lipoprotein modification by lipases, and lipid transfer activities in type 2 diabetic patients. Diabetologia 49: 527-537, 2006

40. Corey KE, Vuppalanchi R, Wilson LA, Cummings OW and Chalasani N: NASH resolution is associated with improvements in HDL and triglyceride levels but not improvement in LDL or non-HDL-C levels. Aliment Pharmacol Ther 41: 301-309, 2015.

41. Zaitone SA, Barakat BM, Bilasy SE, Fawzy MS, Abdelaziz EZ and Farag NE: Protective effect of boswellic acids versus pioglitazone in a rat model of diet-induced non-alcoholic fatty liver disease: Influence on insulin resistance and energy expenditure. Naunyn Schmiedebergs Arch Pharmacol 388: 587-600, 2015

42. Djaouti L, Jourdan T, Demizieux L, Chevrot M, Gresti J, Vergès B and Degrace P: Different effects of pioglitazone and rosiglitazone on lipid metabolism in mouse cultured liver explants. Diabetes Metab Res Rev 26: 297-305, 2010.

43. LeBrasseur NK, Kelly M, Tsao TS, Farmer SR, Saha AK, Ruderman NB and Tomas E: Thiazolidinediones can rapidly activate AMP-activated protein kinase in mammalian tissues. 75-E181. Am J Physiol Endocrinol Metab 291: E175-E181, 2006.

44. Saha AK, Avilucea PR, Ye JM, Assifi MM, Kraegen EW and Ruderman NB: Pioglitazone treatment activates AMP-activated protein kinase in rat liver and adipose tissue in vivo. Biochem Biophys Res Commun 314: 580-585, 2004.

45. Banini BA and Sanyal AJ: Current and future pharmacologic treatment of nonalcoholic steatohepatitis. Curr Opin Gastroenterol 33: 134-141, 2017.

46. Mohan SK, Veeraraghavan VP and Jainu M: Effect of pioglitazone, quercetin and hydroxy citric acid on extracellular matrix components in experimentally induced non-alcoholic steatohepatitis. Iran J Basic Med Sci 18: 832-836, 2015.

47. Steppan CM, Bailey ST, Bhat S, Brown EJ, Banerjee RR, Wright CM, Patel HR, Ahima RS and Lazar MA: The human Resistin links obesity to diabetes. Nature 409: 307-312, 2001.

48. Jamaluddin MS, Weakley SM, Yao Q and Chen C: Resistin: functional roles and therapeutic considerations for cardiovascular disease. Br J Pharmacol 165: 622-632, 2012.

49. Kubota N, Terauchi Y, Kubota T, Kumagai H, Itoh S, Satoh H, Yano W, Ogata H, Tokuyama K, Takamoto I, et al: Pioglitazone ameliorates insulin resistance and diabetes by both adiponectin-dependent and -independent pathways. J Biol Chem 281: 8748-8755, 2006

50. Dongiovanni P, Rametta R, Meroni M and Valenti L: The role of insulin resistance in nonalcoholic steatohepatitis and liver disease development-a potential therapeutic target? Expert Rev Gastroenterol Hepatol 10: 229-242, 2016.

51. Bajaj M, Suraamornku S, Hardies LJ, Pratipanawatr T and DeFronzo RA: Plasma resistin concentration, hepatic fat content, and hepatic and peripheral insulin resistance in pioglitazone-treated type II diabetic patients. Int J Obes Relat Metab Disord 28: 783-789, 2004.

52. Park HK and Ahima RS: Physiology of leptin: Energy homeostasis, neuroendocrine function and metabolism. Metabolism 64: 24-34, 2015.

53. Cedernaes J, Alsiö J, Västermark A, Risérus U and Schiöth HB: Adipose tissue stearoyl-CoA desaturase 1 index is increased and linoleic acid is decreased in obesity-prone rats fed a high-fat diet. Lipids Health Dis 12: 2, 2013. 
54. Amitani M, Asakawa A, Amitani $\mathrm{H}$ and Inui A: The role of leptin in the control of insulin-glucose axis. Front Neurosci 7: 51, 2013

55. Zhou Y and Rui L: Leptin signaling and leptin resistance: Front Med 7: 207-222, 2013

56. Huang XD, Fan Y, Zhang H, Wang P, Yuan JP, Li MJ and Zhan XY: Serum leptin and soluble leptin receptor in non-alcoholic fatty liver disease. World J Gastroenterol 14 2888-2893, 2008

57. Canbakan B, Tahan V, Balci H, Hatemi I, Erer B, Ozbay G, Sut N, Hacibekiroglu M, Imeryuz N and Senturk H: Leptin in nonalcoholic fatty liver disease. Ann Hepatol 7: 249-254, 2008.

58. Cohen P, Ntambi JM and Friedman JM: Stearoyl-CoA desaturase-1 and the metabolic syndrome. Curr Drug Targets Immune Endocr Metabol Disord 3: 271-280, 2003.

59. D'souza AM, Neumann UH, Glavas MM and Kieffer TJ: The glucoregulatory actions of leptin. Mol Metab 6: 1052-1065, 2017.

60. He J, Xu C, Kuang J, Liu Q, Jiang H, Mo L, Geng B and Xu G: Thiazolidinediones attenuate lipolysis and ameliorate dexamethasone-induced insulin resistance. Metabolism 64: 826-836, 2015

61. Adams LA, Anstee QM, Tilg H and Targher G: Non-alcoholic fatty liver disease and its relationship with cardiovascular disease and other extrahepatic diseases. Gut 66: 1138-1153, 2017.

62. Dobrzyn P, Ntambi JM and Dobrzyn A: Stearoyl-CoA desaturase: A novel control point of lipid metabolism and insulin sensitivity. Eur J Lipid Sci Technol 110: 93-100, 2008

63. Heinemann FS and Ozols J: Stearoyl-CoA desaturase, a short-lived protein of endoplasmic reticulum with multiple control mechanisms. Prostaglandins Leukot. Essent Fatty Acids 68: 122-133, 2003.

64. Chong MF, Hodson L, Bickerton AS, Roberts R, Neville M, Karpe F, Frayn KN and Fielding BA: Parallel activation of de novo lipogenesis and stearoyl-CoA desaturase activity after $3 \mathrm{~d}$ of high-carbohydrate feeding. Am J Clin Nutr 87: 817-823, 2008.

65. Cohen P and Friedman JM: Leptin and the control of metabolism: Role for stearoyl-CoA desaturase-1(SCD-1). J Nutr 134: 2455S-2463S, 2004

66. Dobrzyn A and Ntambi JM: The role of stearoyl-CoA desaturase in body weight regulation. Trends Cardiovasc Med 14: 77-81, 2004.

67. Dobrzyn P, Jazurek M and Dobrzyn A: Stearoyl-CoA desaturase and insulin signaling-what is the molecular switch? Biochim Biophys Acta 1797: 1189-1194, 2010.

68. Von Loeffelholz C, Döcke S, Lock JF, Lieske S, Horn P, Kriebel J, Wahl S, Singmann P, de Las Heras Gala T, Grallert $\mathrm{H}$, et al: Increased lipogenesis in spite of upregulated hepatic 5'AMP-activated protein kinase in human non-alcoholic fatty liver. Hepatol Res 47: 890-901, 2017.

69. Lee JJ, Lambert JE, Hovhannisyan Y, Ramos-Roman MA, Trombold JR, Wagner DA and Parks EJ: Palmitoleic acid is elevated in fatty liver disease and reflects hepatic lipogenesis. Am J Clin Nutr 101: 34-43, 2015.

70. Gaggini M, Cabiati M, Del Turco S, Navarra T, De Simone P, Filipponi F, Del Ry S, Gastaldelli A and Basta G: Increased FNDC5/Irisin expression in human hepatocellular carcinoma. Peptides 88: 62-66, 2017.

71. Mock K, Lateef S, Benedito VA and Tou JC: High-fructose corn syrup-55 consumption alters hepatic lipid metabolism and promotes triglyceride accumulation. J Nutr Biochem 39: 32-39, 2017.

72. Liu L, Wang S, Yao L, Li JX, Ma P, Jiang LR, Ke DZ, Pan YQ and Wang JW: Long-term fructose consumption prolongs hepatic stearoyl-CoA desaturase 1 activity independent of upstream regulation in rats. Biochem Biophys Res Commun 479: 643-648, 2016.

73. Hulver MW, Berggren JR, Carper MJ, Miyazaki M, Ntambi JM, Hoffman EP, Thyfault JP, Stevens R, Dohm GL, Houmard JA, et al: Elevated stearoyl-CoA desaturase-1 expression in skeletal muscle contributes to abnormal fatty acid partitioning in obese humans. Cell Metabolism 2: 251-261, 2005.
74. Hellemans KH, Hannaert JC, Denys B, Steffensen KR, Raemdonck C, Martens GA, Van Veldhoven PP, Gustafsson JA and Pipeleers D: Susceptibility of pancreatic beta cells to fatty acids is regulated by LXR/PPAR $\alpha$-dependent stearoyl-coenzyme a desaturase. PLoS One 4: e7266, 2009.

75. Chiu S, Mulligan K and Schwarz JM: Dietary carbohydrates and atty liver disease: De novo lipogenesis. Curr Opin Clin Nutr Metab Care 21: 277-282, 2018.

76. Softic S, Cohen DE and Kahn CR: Role of dietary fructose and hepatic de novo Lipogenesis in fatty liver disease. Dig Dis Sci 61: 1282-1293, 2016

77. Silbernagel G, Kovarova M, Cegan A, Machann J, Schick F, Lehmann R, Häring HU, Stefan N, Schleicher E, Fritsche A and Peter A: High hepatic SCD1 activity is associated with low liver fat content in healthy subjects under a lipogenic diet. J Clin Endocrinol Metab 97: E2288-E2292, 2012.

78. Kurebayashi S, Hirose T, Miyashita Y, Kasayama S and Kishimoto T: Thiazolidinediones downregulate stearoyl-CoA desaturase 1 gene expression in 3T3-L1 adipocytes. Diabetes 46 2115-2118, 1997

79. Sun S, Zhang Z, Pokrovskaia N, Chowdhury S, Jia Q, Chang E, Khakh K, Kwan R, McLaren DG, Radomski CC, et al: Discovery of triazolone derivatives as novel, potent stearoyl-CoA desaturase-1 (SCD1) inhibitors. Bioorg Med Chem 23: 455-465, 2015.

80. Meshkani R, Sadeghi A, Taheripak G, Zarghooni M, Gerayesh-Nejad S and Bakhtiyari S: Rosiglitazone, a PPAR $\gamma$ agonist, ameliorates palmitate-induced insulin resistance and apoptosis in skeletal muscle cells. Cell Biochem Funct 32: 683-691, 2014.

81. Ikeda J, Ichiki T, Takahara Y, Kojima H, Sankoda C, Kitamoto S, Tokunou $\mathrm{T}$ and Sunagawa K: PPAR $\gamma$ agonists attenuate palmitate-induced ER stress through up-regulation of SCD-1 in macrophages. PLoS One 10: e0128546, 2015.

82. Risérus U, Tan GD, Fielding BA, Neville MJ, Currie J, Savage DB, Chatterjee VK, Frayn KN, O'Rahilly S and Karpe F: Rosiglitazone increases indexes of stearoyl-CoA desaturase activity in humans: Link to insulin sensitization and the role of dominant-negative mutation in peroxisome proliferator-activated receptor-gamma. Diabetes 54: 1379-1384, 2005.

83. Leekumjorn S, Cho HJ, Wu Y, Wright NT, Sum AK and Chan C: The role of fatty acid unsaturation in minimizing biophysical changes on the structure and local effects of bilayer membranes. Biochim Biophys Acta 1788: 1508-1516, 2009.

84. Leamy AK, Egnatchik RA and Young JD: Molecular mechanisms and the role of saturated fatty acids in the progression of non-alcoholic fatty liver disease. Prog Lipid Res 52: 165-174, 2013.

85. Arisqueta L, Navarro-Imaz H, Rueda Y and Fresnedo O Cholesterol mobilization from hepatic lipid droplets during endotoxemia is altered in obese ob/ob mice. J Biochem 158: 321-329, 2015

86. Saliani N, Darabi M, Yousefi B, Baradaran B, Khaniani MS, Darabi M, Shaaker M, Mehdizadeh A, Naji T and Hashemi M: PPAR $\gamma$ agonist-induced alterations in $\Delta 6$-desaturase and stearoyl-CoA desaturase 1: Role of MEK/ERK1/2 pathway. World J Hepatol 5: 220-225, 2013.

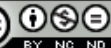

This work is licensed under a Creative Commons Attribution-NonCommercial-NoDerivatives 4.0 International (CC BY-NC-ND 4.0) License. 